\title{
Increase in buckling loads of plates by introduction of cutouts
}

Received: 30 October 2018 / Revised: 5 March 2019 / Published online: 24 May 2019

(C) The Author(s) 2019

\begin{abstract}
It might seem amazing: Cutting a hole in a plate can increase the buckling strength! It is the objective of this paper to present and clarify this astounding phenomenon. In lightweight design, typically thin-walled structures are used. Therefore, buckling must be considered as a possible failure mode. One might assume that removing material, and thus, reducing stiffness must result in a reduction of the buckling strength. However, perhaps surprisingly, it can be shown that introduction of cutouts, placed appropriately, can under certain conjunctures increase buckling loads. At the same time, the structural mass is reduced. Thus, the paper presents a measure, which can be used for fulfillment of a requirement in lightweight design in a twofold manner: increase in buckling strength by reduction of mass! In addition to describing a nice theoretical peculiarity, it might be of more importance from the engineering point of view that the presented methodology may help designers of lightweight structures, e.g., for aerospace applications, to place openings, which are required for some reasons in a plate being part of the construction, at such positions, at which the plate's buckling resistance is just slightly or not at all reduced or even increased, and to avoid placing holes in unfavorable areas. Based on the Rayleigh-Ritz method in terms of the Rayleigh quotient, criteria and procedures are derived which can be used to find beneficial positions for cutouts and such ones, at which cutouts should not be placed.
\end{abstract}

\section{Introduction}

It is common practice in lightweight design of metallic structures to use assemblies of thin-walled skins reinforced by stiffeners or, in reverse, skins reinforcing beam frame structures. This way, quite often, thin plates act as shear webs [1]. In such situations, the instant of elastic buckling does by far not coincide with structural failure. Because of the stable character of the post-buckling behavior, quite large reserves of loadcarrying capacity may exist [2]. Nevertheless, the appearance of buckling patterns is in most cases undesirable. Hence, the design for usual load cases must be buckling resistant.

It is a quite common opinion that removing material, and thus, reducing stiffness results in a reduction of the buckling strength. This engineering experience gets, with respect to buckling of plates with cutouts, manifested in several papers; see, e.g., [3-8] and papers cited therein. (A quite intensive search has brought one rather old paper to light, namely [9], in which, based on parametrized finite element studies, an indication is given that in special situations the critical load may increase when an opening is cut in a plate.) The, in general, expected negative effect of cutouts is even more pronounced when the cutouts are large enough to

J. Blesa Gracia

RUAG Aerospace Structures GmbH, Gilching, Germany

E-mail: info.de.aerostructures@ ruag.com

F. G. Rammerstorfer ( $\varangle)$

Vienna University of Technology (TU Wien), Vienna, Austria

E-mail: franz.rammerstorfer@tuwien.ac.at 
effectuate local buckling. Such local instabilities are the reason why the critical tensile load for buckling of stretched plates is drastically reduced even by relatively small cutouts, see [10].

Thus, it might be surprising that, under certain conjunctures, the introduction of cutouts of proper shape and size, placed appropriately, can increase the buckling resistance of plates under in-plane loading. At the same time, the cutouts lead to a reduction of the structural mass. Obviously, there is a measure which can be in a twofold manner advantageous with respect to the requirements in lightweight design; it increases the buckling strength by reduction of mass! It is not that much out of the ordinary that in special cases removing material does not only reduce mass but also improves structural performance. For instance, proper material removal in terms of undercuts in notched regions of structural elements or at positions with singular stress fields, as, e.g., at crack tips or the surface of structures with bi-material interfaces, increases the lifetime under cyclic loading; see, for instance, [11]. However, the fact that cutting holes in a plate may increase its buckling resistance appears to be remarkable.

\section{Methodology and criteria}

\subsection{The Rayleigh quotient}

It is a common procedure for calculating approximate values of buckling loads to use a single-term Ritz-ansatz for the buckling mode in conjunction with the so-called Rayleigh quotient, also called Rayleigh-Ritz quotient. For buckling problems under consideration in this paper, the Rayleigh quotient is derived under in-plane loading on the basis of the linearized v. Kármán's plate equation for rectangular plates (with the mid-surface in the in the $x-y$ plane), made of isotropic, homogeneous, linear elastic material, and loaded by in-plane edge forces per unit length, $N_{i j}$, only,

$$
K \Delta \Delta w-n_{x x} w_{, x x}-n_{y y} w_{, y y}-2 n_{x y} w_{, x y}=0 .
$$

In this equation and in all of the following considerations, the quantities $n_{i j}(x, y)$ represent the distribution of the membrane forces (in-plane stress resultants per unit length), with $n_{x x}$ and $n_{y y}$ as well as $N_{x x}$ and $N_{y y}$ being positive when compressive. Also the sign of $n_{x y}$ as well as that of $N_{x y}$ is assumed to be just opposite to the classical convention. This means, e.g., that an external edge shear load $N_{x y}$, acting in $y$ direction along the edge, of which the outward oriented normal vector points to the $x$ direction, is not positive but negative.

The bending stiffness $K$ of the plate is given by $K=E t^{3} /\left[12\left(1-v^{2}\right)\right]$, with Young's modulus $E$, Poisson's ratio $v$, and plate thickness $t$, and $w(x, y)$ the transversal displacement.

As shown in several textbooks (e.g., [12-14]), the Rayleigh quotient for estimating fundamental eigenfrequencies or buckling loads is derived from energy principles by approximating the respective mode $\hat{q} \hat{\phi}(x, y)$ by a single-term Ritz-ansatz, which has to fulfill at least the essential, i.e., kinematic boundary conditions, $q \phi(x, y) \approx \hat{q} \hat{\phi}(x, y)$. The Rayleigh quotient represents an upper bound both for the fundamental natural frequency and for the buckling load, respectively. Thus, in [15], one finds the notation "Rayleigh's inequality." Provided that no self-equilibrating stresses (e.g., residual stresses, thermal stresses, etc.) exist, in buckling analyses it approximates the buckling load factor $\hat{\lambda}$, by which the nominal loading $N_{i j}$ and, consequently, the corresponding membrane stress field $n_{i j}(x, y)$ has to be multiplied in order to bring the plate to buckling. For rectangular plates, having their edges along $x=0$ and $x=a$ as well as $y=0$ and $y=b$, respectively, under in-plane edge loading $N_{i j}$ and with exclusion of eigenstresses, i.e., not being caused by external mechanical loads (e.g., residual stresses or thermal stresses), the Rayleigh quotient leads to the estimated critical load multiplier,

$$
\lambda=\frac{K \int_{S}\left[\Delta \phi \Delta \phi-2(1-v)\left(\phi_{, x x} \phi_{, y y}-\phi_{, x y} \phi_{, x y}\right] \mathrm{d} S\right.}{\int_{S} n_{i j} \phi_{, i} \phi_{, j} \mathrm{~d} S} \geq \hat{\lambda} .
$$

For rectangular plates with all edges immovable in $z$ direction, the application of the Gauss-Bonnet theorem leads to the fact that the contribution of $2(1-v)\left(\phi_{, x x} \phi_{, y y}-\phi_{, x y} \phi_{, x y}\right)$ to the integral in the numerator of Eq. (2) disappears, and the Rayleigh quotient reads then

$$
\lambda=\frac{K \int_{S} \Delta \phi \Delta \phi \mathrm{d} S}{\int_{S} n_{i j} \phi_{, i} \phi_{, j} \mathrm{~d} S} \geq \hat{\lambda} .
$$


This expression can also be found in [16].

As long as no cutout is in the plate, i.e., for the unperforated plate, and only uniformly distributed in-plane edge loads are acting, it is obvious that for rectangular plates with all edges simply supported or clamped but moveable in the plate's mid-surface, the homogeneously distributed membrane forces $n_{i j}$ can be replaced by $N_{i j}$, leading to

$$
\lambda=\frac{K \int_{S} \Delta \phi \Delta \phi \mathrm{d} S}{\int_{S} N_{i j} \phi_{, i} \phi, j \mathrm{~d} S} \geq \hat{\lambda} .
$$

The integration domain $S$ is the mid-surface area of the plate. Of course, the better the shape function $\phi(x, y)$ (which at least must fulfill the kinematic boundary conditions) approximates the fundamental buckling mode, i.e., the more $\phi(x, y) \propto \hat{\phi}(x, y)=\hat{\phi}_{1}(x, y)$, the closer is the estimated critical load multiplier $\lambda$ to the "exact" value $\hat{\lambda}=\hat{\lambda}_{1}$. When no analytical solution is available, a suitable finite element (FE) model, i.e., elements of high quality in a sufficiently fine mesh, can be used in a linear buckling eigenvalue analysis, from which the smallest eigenvalue can be accepted as $\hat{\lambda}$, and the corresponding eigenvector, together with the elements' displacement interpolation functions, represents quite well $\hat{\phi}(x, y)$.

In "standard" load cases, i.e., uniaxial compressive edge loading in $x$ or in $y$ direction is applied with intensity 1 force unit per unit length, or pure shear loading with intensity +1 or -1 , all uniformly distributed along the corresponding edges, the Rayleigh quotient renders directly an upper bound for the corresponding critical load intensity, i.e., the buckling load multiplier for such a nominal loading.

\subsection{Effect of the introduction of cutouts}

Cutting a hole (or holes) in the plate and applying the same uniformly distributed edge loading $N_{i j}$ and the same boundary conditions as for the unperforated plate (i.e., the plate without any cutout) modifies not only the integration domain $S$ in Eq. (2), which for the perforated plate is now denoted by $S^{*}$, but also the membrane force distribution, which becomes inhomogeneous; it is called $n_{i j}^{*}(x, y)$. Needless to say that the buckling load is changed to become $\hat{\lambda}^{*}$, and an altered buckling mode $\hat{\phi}_{1}^{*}$ appears; compare, e.g., Figs. 1 and 2. Hence, in Eq. (2) $\phi$ is to be replaced by a modified shape function $\phi^{*}$, which better approximates the altered buckling mode $\hat{\phi}_{1}^{*}$. Furthermore, at the boundary of the cutout(s) the condition $w=0$ must no longer be assumed, and the edges of the cutouts are not necessarily straight lines. Therefore, the Gauss-Bonnet theorem does no longer lead to a disappearance of the second term of the integrand in the numerator of Eq. (2), and the Rayleigh quotient for the perforated plate reads

$$
\lambda^{*}=\frac{K \int_{S^{*}}\left[\Delta \phi^{*} \Delta \phi^{*}-2(1-v)\left(\phi_{, x x}^{*} \phi_{, y y}^{*}-\phi_{, x y}^{*} \phi_{, x y}^{*}\right)\right] \mathrm{d} S}{\int_{S^{*}} n_{i j}^{*} \phi_{, i}^{*} \phi_{, j}^{*} \mathrm{~d} S} \geq \hat{\lambda}^{*}
$$

Let us, for the sake of simplicity but without restriction of generality, assume that only one hole is cut. Its region $S^{* *}$ is assumed to be completely in the interior of $S$, i.e., $S^{* *} \subset S$, and the remaining area of the plate is

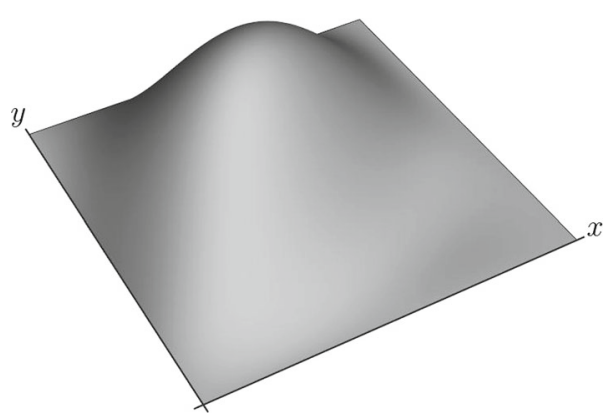

Fig. 1 Buckling mode of a purely shear loaded square plate with CCSS boundary conditions (FE simulation) 


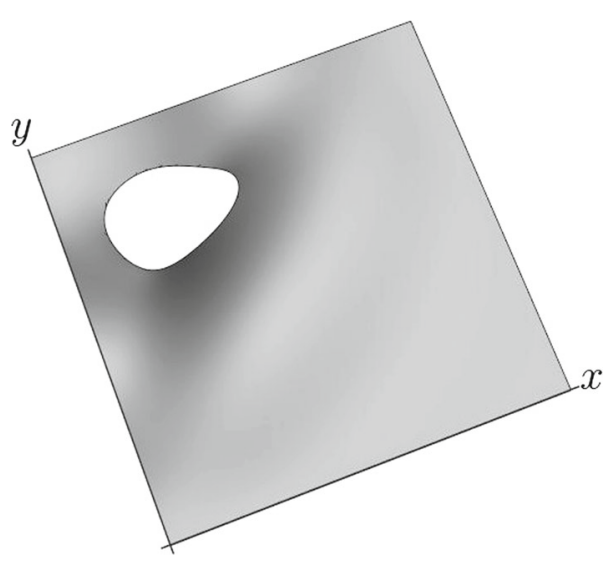

Fig. 2 The same plate now with an elliptical cutout; $\varphi=1.134$, i.e., $\lambda^{*}$ is increased by $13.4 \%$

$S^{*}=S \backslash S^{* *}$. Furthermore, it is assumed that the cutout is not too large and is located sufficiently distant from the plate's edges in order to ensure that local buckling does not appear.

With

$$
\varphi=\frac{\lambda^{*}}{\lambda}
$$

the effect of the cutout on the buckling load is quantified as the ratio between the buckling load multiplier of the plate with cutout, i.e., $\lambda^{*}$, and without cutout, i.e., $\lambda$, respectively.

Again, the ratio $\varphi$ gets the exact value $\hat{\varphi}$ only if the true mode shapes, $\hat{\phi}$ and $\hat{\phi}^{*}$, are used instead of $\phi$ and $\phi^{*}$. By experience, one might expect $\varphi<1$ in any case. However, the following derivations will show that there are possibilities for $\varphi>1$.

Remark 1 In "Appendix A", it is shown that $n_{i j}^{*}$ in Eq. (5) can be replaced by $N_{i j}$.

Remark 2 As long as isotropic, linear elastic material with a fixed Poisson's ratio $v$ is assumed (in this paper $v=0.25$ is chosen) and linear buckling is concerned, here and in the following, the findings derived from the above derivations can be considered like being stated in dimensionless form. This means that the effect of cutouts with respect to the change in the buckling load, expressed by the ratio $\varphi$, does neither depend on actual material parameters nor on absolute values of geometrical dimensions. Only geometrical proportions of the mid-surface of the plate enters the determination of the ratio $\varphi$.

Remark 3 The equation in the inequality (5) can be used in an optimization algorithm, where positions as well as the geometric parameters of cutouts, required by the use of the structural plate under consideration, should be determined as optimization parameters for maximizing the objective function $\varphi$. With the inclusion of the search for the single-term shape function, expressed by a function with a number of free parameters, approximating best the buckling mode, i.e., leading to the minimum of the estimated buckling load in each optimization step, the approach would lead to a max-min-problem similar to that described in [17]. Since it is the intention of the present paper to describe the fundamental ideas, the optimization is out of its scope. It has been left for future elaborations.

\subsection{Boundary conditions and an illustrative example}

In this paper, the boundary conditions of the considered rectangular plates are described by a sequence of four of the capital letters $\mathrm{C}$ and $\mathrm{S}$, respectively, each letter for an edge, starting with the edge placed along the $x$-axis, and the boundary conditions at the other edges are described contiguously in an anticlockwise sequence. The letter $\mathrm{C}$ denotes the boundary conditions for edges clamped but moveable in the $x-y$ plane, and $\mathrm{S}$ stands for simply supported edges, i.e., hinged and in-plane movable.

As an example for illustrating the increase in the critical load multiplier by introduction of a cutout compare between Figs. 1 and 2, showing buckling of a square plate with CCSS boundary conditions, loaded by uniformly distributed edge shear forces. Here and in the following Figures, the presented buckling modes and the values for $\varphi$ in the Figure captions result from FE-simulations. 


\section{An analytical approach for finding appropriate positions for cutouts}

Let us accomplish a gedankenexperiment: We consider a plate in which we cut a single tiny virtual circular hole at various positions, just for sensing at which positions the hole would show a larger or smaller effect with respect to increase in the buckling resistance of the plate. We call this virtual hole "sensor hole." Because our sensor hole is quite tiny, we may neglect the difference between $\hat{\phi}$ and $\hat{\phi}^{*}$ and, consequently, also the difference between $\phi$ and $\phi^{*}$. Furthermore, let us take advantage of the fact mentioned in the above Remark 1 .

Assuming Remark 1, one can write:

$$
\varphi=\frac{\lambda^{*}}{\lambda}=\frac{\frac{K \int_{S^{*}}\left[\Delta \phi^{*} \Delta \phi^{*}-2(1-v)\left(\phi_{, x x}^{*} \phi_{, y}^{*}-\phi_{, x y}^{*} \phi_{, x y}^{*}\right] \mathrm{d} S\right.}{\int_{S^{*}} N_{i j} \phi_{, i}^{*} \phi_{, j}^{*} \mathrm{~d} S}}{\frac{K \int_{S} \Delta \phi \Delta \phi \mathrm{d} S}{\int_{S} N_{i j} \phi_{, i} \phi, j} \mathrm{~d} S}
$$

Substituting $\phi^{*}$ with $\phi$ in the previous equation, splitting the integration domain $\mathrm{S}$ into the regions $S=$ $S^{*} \cup S^{* *}$, where $S^{* *}$ is the region of the sensor hole, hence, $S^{*}=S \backslash S^{* *}$, and using the integral properties, after some algebraic manipulation, we obtain:

$$
\varphi=1+\frac{\int_{S^{* *}} N_{i j} \phi_{, i} \phi_{, j} \mathrm{~d} S \cdot \int_{S} \Delta \phi \Delta \phi \mathrm{d} S-\int_{S} N_{i j} \phi_{, i} \phi_{, j} \mathrm{~d} S \cdot \int_{S^{* *}}\left[\Delta \phi \Delta \phi-2(1-v)\left(\phi_{, x x} \phi_{, y y}-\phi_{, x y} \phi_{, x y}\right)\right] \mathrm{d} S}{\int_{S^{*}} N_{i j} \phi_{, i} \phi_{, j} \mathrm{~d} S \cdot \int_{S} \Delta \phi \Delta \phi \mathrm{d} S}
$$

where the integrals over $S^{* *}$, appearing in (8) and later on, are to be understood as the differences between the corresponding integrals over $S$ and over $S^{*}$.

At least for pure compression with uniformly distributed edge compression loads, i.e., $N_{x y}=0$, it is obvious that the numerator in the second term controls whether the buckling loading increases or decreases with the hole. If the numerator is positive, then $\lambda^{*}>\lambda$. With the definition

$$
\gamma=\frac{\int_{S} \Delta \phi \Delta \phi \mathrm{d} S}{\int_{S} N_{i j} \phi_{, i} \phi_{, j} \mathrm{~d} S} / \frac{\int_{S^{* *}}\left[\Delta \phi \Delta \phi-2(1-v)\left(\phi_{, x x} \phi_{, y y}-\phi_{, x y} \phi_{, x y}\right)\right] \mathrm{d} S}{\int_{S^{* *}} N_{i j} \phi_{, i} \phi_{, j} \mathrm{~d} S}
$$

this requirement leads, under the accepted assumptions, to

$$
\gamma>1 \Longleftrightarrow \lambda^{*}>\lambda
$$

Let us now introduce the following abbreviations,

$$
\begin{aligned}
& \Psi(x, y)=\Delta \phi \Delta \phi-2(1-v)\left(\phi_{, x x} \phi_{, y y}-\phi_{, x y} \phi_{, x y}\right), \\
& \Sigma(x, y)=N_{i j} \phi_{, i} \phi_{, j} .
\end{aligned}
$$

From the criterion according to Eq. (10), together with the definition (9), one can conclude that the sensor hole will indicate positions with small $\Psi(x, y)$ values and large $\Sigma(x, y)$ values as locations for (not too large) real cutouts offering the chance to increase the buckling resistance. In contrast, a decrease in the buckling load must be expected if the cutout is located in an area with large values of $\Psi$ and small $\Sigma$ values.

So far, in order to ensure a positive denominator in Eq. (8), it was assumed that no shear load is acting, i.e., $N_{x y}=0$. If, however, uniformly distributed shear loading along the edges is involved, too, one has to check the sign of the contributions of $N_{x y} \phi_{, x} \phi_{, y}$ and $N_{y x} \phi_{, y} \phi_{, x}$ to the integrals $\int N_{i j} \phi_{, i} \phi_{, j} \mathrm{~d} S$ over the domains $S$ and $S^{* *}$, respectively. Only if both integrals, including the contributions due to shear loading in their integrand, are positive or negative, then condition (10) with the definition according to Eq. (9) holds further on.

These qualitative measures should now become more quantitative by the following derivations. The simplifying assumption, made for the gedankenexperiment, becomes more and more accurate, if the radius of the sensor hole approaches zero. Finally, the denominator in Eq. (9) for vanishing size of $S^{* *}$, that is,

$$
\kappa=\lim _{S^{* *} \rightarrow 0} \frac{\int_{S^{* *}} \Psi(x, y) \mathrm{d} S}{\int_{S^{* *}} \Sigma(x, y) \mathrm{d} S},
$$


indicates quantitatively the appropriateness of positions for real cutouts. Using l'Hospital's rule, Eq. (13) renders

$$
\kappa(x, y)=\frac{\Psi(x, y)}{\Sigma(x, y)} .
$$

Eventually, areas with

$$
\hat{\gamma}(x, y)=\frac{1}{\kappa(x, y)} \frac{\int_{S} \Delta \phi \Delta \phi \mathrm{d} S}{\int_{S} N_{i j} \phi_{, i} \phi, j \mathrm{~d} S}>1
$$

are potentially appropriate for the introduction of real cutouts, and the larger the value of $\hat{\gamma}(x, y)$, the more are such areas appropriate.

It is quite obvious that areas, in which $\hat{\gamma}(x, y)$ is much smaller than 1.0, are prone to provide positions for cutouts which reduce the buckling resistance. Examples for this disadvantageous effect of cutouts are discussed in Sect. 4.

\subsection{Simply supported square plate under uniaxial compression}

In order to demonstrate the use of the above idea, consider a square plate with edge length $a$ under uniaxial compression in $x$ direction. All edges are simply supported (SSSS boundary conditions). For the unperforated configuration, the fundamental buckling mode is known as

$$
\phi(x, y)=\sin \left(\pi \frac{x}{a}\right) \sin \left(\pi \frac{y}{a}\right) .
$$

Figures 3 and 4 show the distribution of the functions $\Psi(x, y)$ and $N_{x x} \phi_{, x} \phi_{, x}$, respectively (lighter implies larger values of the functions). Having the above in mind, one finds that a hole in the center of the plate (where large $\Psi$ values and small values of $N_{x x} \phi_{, x} \phi_{, x}$ appear) will decrease the buckling load, while in reverse, a hole close to the loaded edge will increase it. Considering now the criterion expressed by Eq. (15), one gets a more precise information from Fig. 5. Again, there and in the following corresponding Figures, areas with large values of $\hat{\gamma}$ are bright while those with low values are dark. Moreover, the depicted white lines correspond to isolines with $\hat{\gamma}(x, y)=1$. Thus, these white lines represent boarders between regions where cutouts have a good chance to increase the buckling resistance and regions in which cutouts should be avoided, if stability is a decisive design criterion. The brighter the areas are, the higher is the chance for increasing the buckling resistance by placing cutouts there. And, vice versa, the darker the area, the more is a weakening effect of cutouts, placed there, to be expected. Figure 5 clearly indicates that for the example under consideration a cutout placed close to the middle of the loaded edge has good chances to lead to an increase in the buckling resistance of the plate.

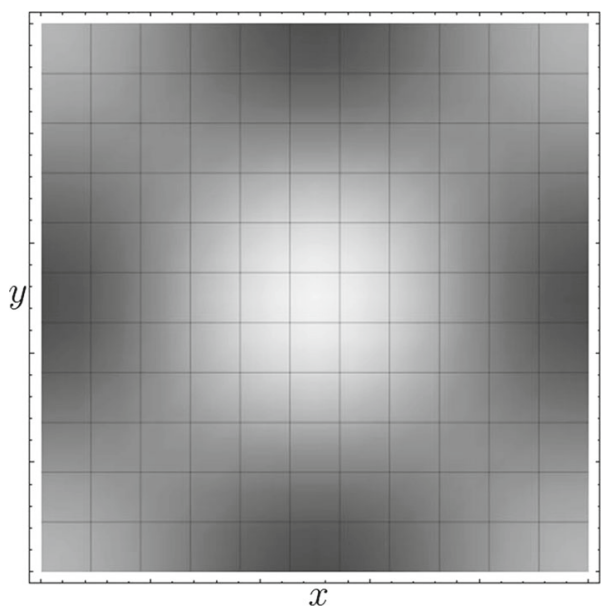

Fig. 3 Distribution of $\Psi(x, y)$ over domain $S$ 


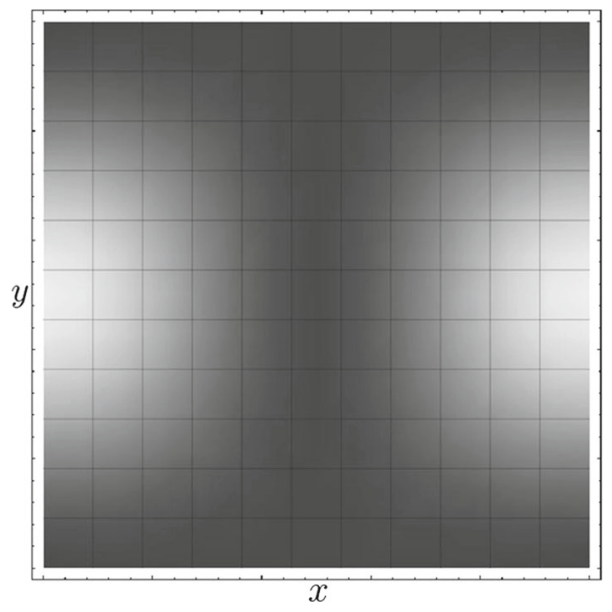

Fig. 4 Distribution of $N_{x x} \phi_{, x} \phi_{, x}$

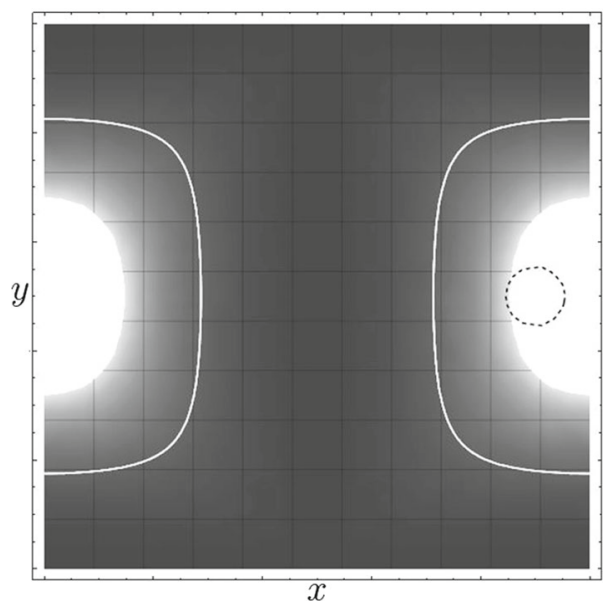

Fig. 5 Distribution of $\hat{\gamma}(x, y)$ over domain $S$

For demonstration purpose, a plate with edge length $a=1$ length unit and circular cutouts of radius $\frac{r}{a}=0.055$ is studied. Three positions of the cutout are considered: Case I: the center of the hole is placed at (dimensionless) coordinates $\left(\frac{x}{a}, \frac{y}{a}\right)$ equal to $(0.5,0.5)$ — centre of the plate; in case II: it is at $(0.9,0.5)($ cutout depicted with a dashed line in Fig. 5), and in case III: at $(0.5,0.1)$, respectively.

The predictions, based on the above considerations, are in accordance with the results of FE calculation of the buckling loads; see Figs. 6, 7, and 8. Because of the small size of the holes, the effect of the cutouts is not very much distinctive. Nevertheless, the results confirm the above statements.

\section{A semi-analytical approach}

If no analytical description of the buckling mode of the unperforated plate is available or if the desired or required cutout(s) shall be relatively large, so that the buckling mode of the unperforated plate differs too much from the buckling mode of the perforated plate, the following procedure is proposed:

(i) Perform a computational buckling analysis of the unperforated plate by solving the eigenvalue problem

$$
\left(\mathbf{K}_{0}+\hat{\lambda} \mathbf{K}_{\mathrm{G}}\right) \boldsymbol{\Phi}=\mathbf{0} .
$$

There, $\mathbf{K}_{0}$ is the stiffness matrix as usually calculated in a linear FE analysis; $\mathbf{K}_{\mathrm{G}}$ is the so-called geometric stiffness matrix (corresponding to 2 nd order theory), calculated under reference loading $N_{i j}$. 


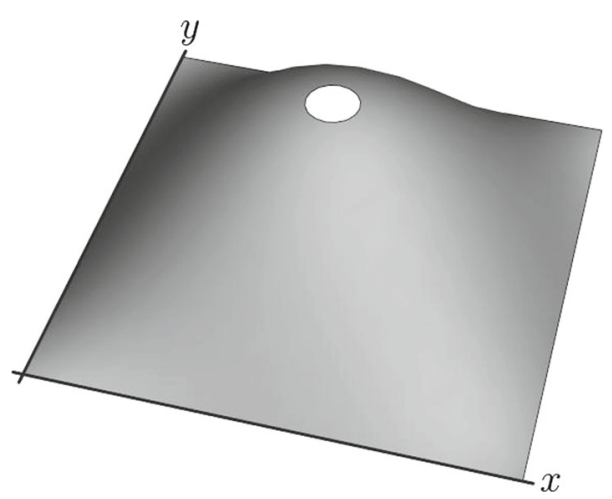

Fig. 6 Hole in the center of the plate; $\varphi=0.953$

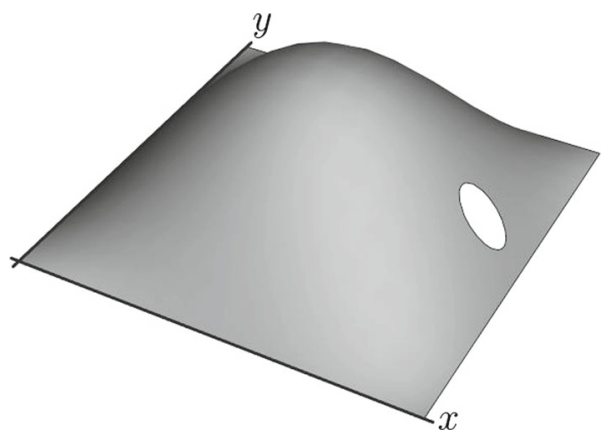

Fig. 7 Hole near a loaded edge; $\varphi=1.006$

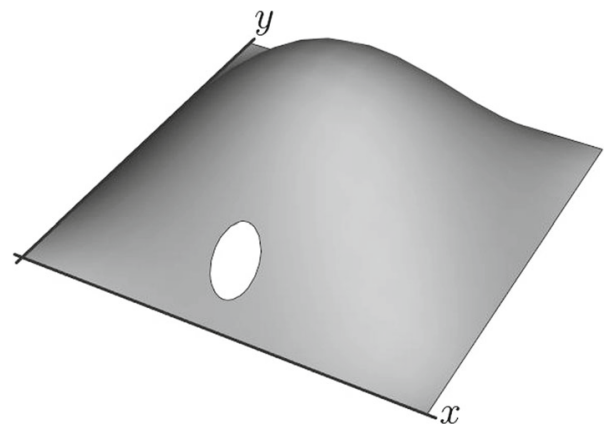

Fig. 8 Hole near an unloaded edge; $\varphi=0.972$

(ii) Construct, for instance by multivariate polynomials (as used in the last example of this paper), a smooth, sufficiently differentiable function $\phi(x, y)$ which approximates the computed buckling mode $\hat{\phi}_{1}$, derived from the eigenvector $\boldsymbol{\Phi}_{1}$, corresponding to the lowest eigenvalue $\hat{\lambda}_{1}$. Then calculate $\Psi(x, y), \Sigma(x, y)$, and $\hat{\gamma}(x, y)$ over the domain $S$.

(iii) Find a region (or regions) in $S$, where $\hat{\gamma}$ is large.

(iv) Place a hole (or holes) in such region(s) and perform a computational buckling analysis with the perforated plate, leading to $\hat{\lambda}^{*}$.

(v) Calculate the ratio $\varphi=\hat{\lambda}_{1}^{*} / \hat{\lambda}_{1}$ and the safe in mass of the plate as $S^{* *} / S \times 100 \%$ for evaluating the efficiency of the cutout(s).

Remark 4 For the sake of comparison between different configurations, the cutouts in the demonstrative examples presented in this Section are of circular shape and have all the same size. This is by far no restriction of generality regarding the shape of the cutouts. 


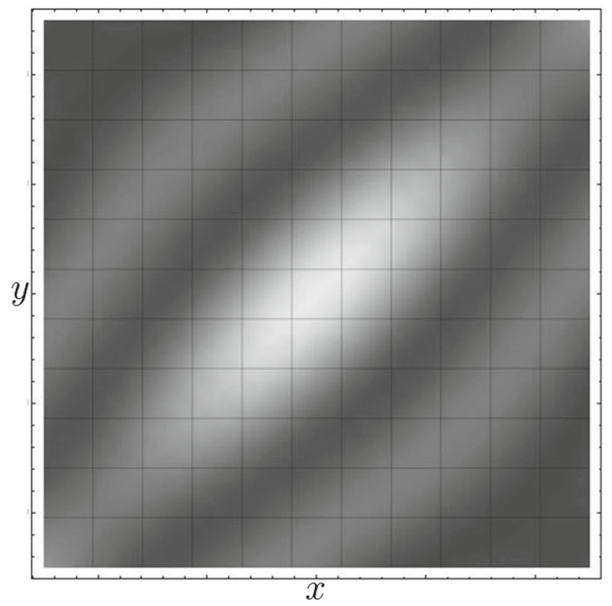

Fig. 9 Function of $\Psi(x, y)$

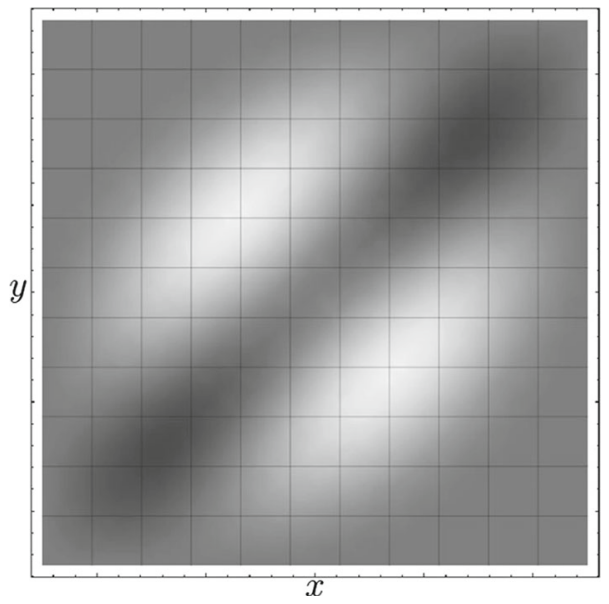

Fig. 10 Function $N_{x y} \phi_{, x} \phi_{, y}$

4.1 Simply supported square plate under pure shear loading

As a still reasonably simple example for the application of the above-described semi-analytical approach let us consider a simply supported square plate (i.e, SSSS boundary conditions) of edge length $2 l$ under pure shear loading $N_{x y}=-1$ force unit by unit length, uniformly distributed along the edges. The following function $\phi(x, y)$ has been found as a suitable Ritz-ansatz for the buckling mode of the unperforated plate:

$$
\phi(x, y)=\frac{\left(x^{2}-l^{2}\right)\left(y^{2}-l^{2}\right)}{l^{4}} \cos ^{2}\left[\left(x \cos \frac{\pi}{4}-y \sin \frac{\pi}{4}\right) \frac{\pi}{l} \cos ^{2} \frac{\pi}{4}\right] .
$$

There, for convenience, the origin of the coordinate system is placed in the middle of the plate area.

With this shape function, $\Psi(x, y), 2 N_{x y} \phi_{, x} \phi, y$, and $\hat{\gamma}(x, y)$ are calculated. Figures 9,10 , and 11 show the distribution of these quantities, providing the information needed to appropriately place a cutout (or cutouts). Finding proper positions for placing cutouts corresponds with making a compromise between choosing a region (or regions) with small $\Psi(x, y)$ and such one(s) with large values of $2 N_{x y} \phi_{, x} \phi_{, y}$. However, it seems to be more convenient to simply look for positions with large values of $\hat{\gamma}(x, y)$. Again, in Fig. 11 the isolines where $\hat{\gamma}(x, y)=1$ are depicted with white lines.

In Fig. 12, the buckling mode of the shear loaded plate with two beneficial circular cutouts, the boundaries of which are shown by black dashed circles in Fig. 11, located in areas of $\hat{\gamma}(x, y)>1$, is presented. The critical load multiplier is increased by about $12 \%$. (By the way, the mass is reduced by around $4 \%$ ). The buckling mode of the same square plate but having two cutouts at unfavorable positions, i.e., $\hat{\gamma}(x, y)<1$, 


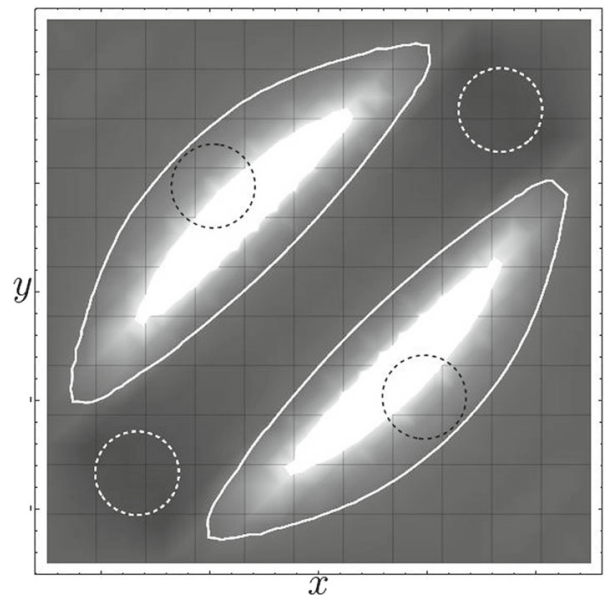

Fig. 11 Function $\hat{\gamma}(x, y)$

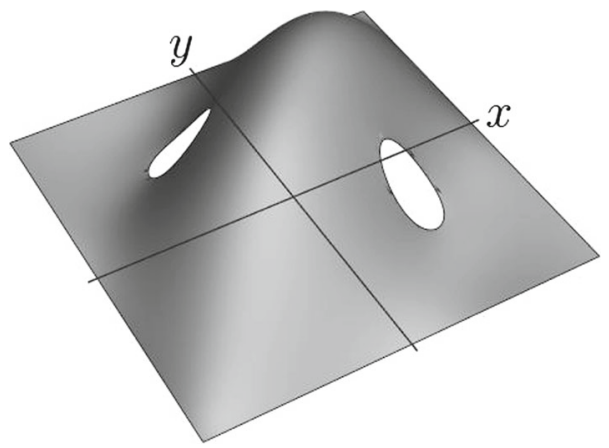

Fig. 12 Buckling mode of the shear loaded square plate with two beneficial holes; $\varphi=1.118$

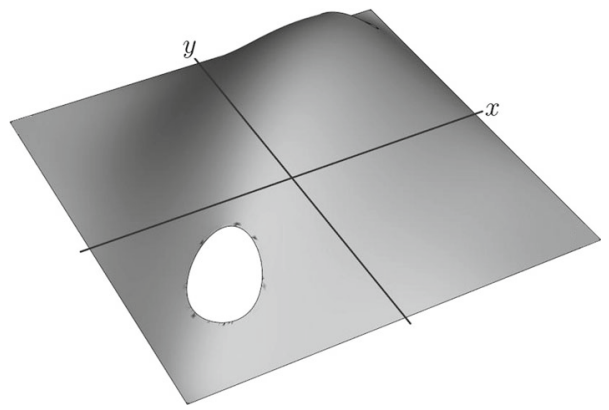

Fig. 13 Buckling mode when two unfavorable holes are in the plate; $\varphi=0.708$

as depicted by the white dotted circles in Fig. 11, is shown in Fig. 13. In this configuration, the critical load multiplier is decreased by about $30 \%$. This large difference between the critical load multipliers of the plate in the described two configurations brings to light the importance of a careful placement if cutouts are required.

This example shows that for plates under shear loading the efficiency of introducing appropriate cutouts for increasing the buckling load is more pronounced than under edge loading in compression. This is because under shear loading the alteration of the buckling mode by introducing cutouts is quite strong. In Fig. 12, one can observe that the introduction of the cutouts "pushes" the out-of-plane deformations in the buckling mode toward the center of the plate. On the other hand, introduction of cutouts in regions with $\hat{\gamma}(x, y)<1$ broadens the buckle; see Fig. 13. 


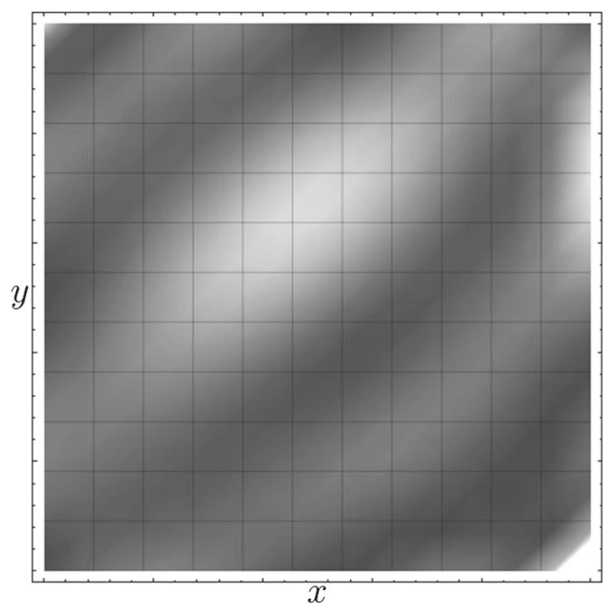

Fig. 14 Square plate, general loading case; $\Psi(x, y)$

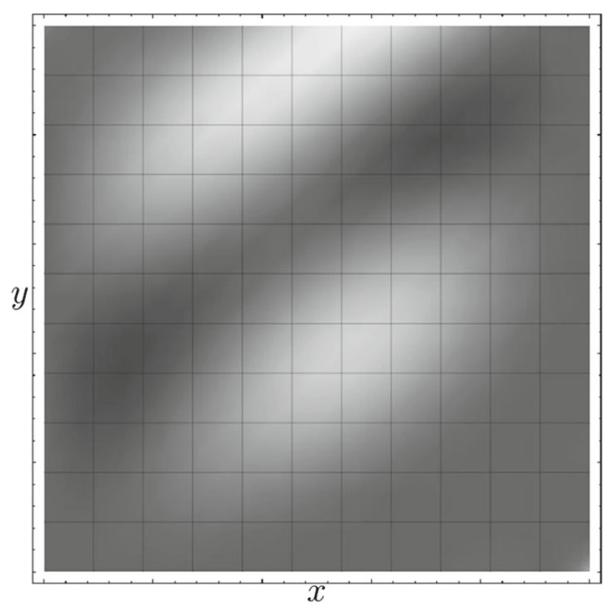

Fig. 15 Square plate, general loading case; $\Sigma(x, y)$

4.2 Square plate under combined loading and mixed boundary conditions

As a last example, a more general case is considered: a square plate under uniaxial unit compression load in $y$ direction, i.e., $N_{y y}=1$ force unit per unit length, combined with unit shear loading $N_{x y}=-1$ force unit per unit length. CCSS boundary conditions are assumed.

The Ritz-ansatz $\phi$, approximating the quite complex fundamental buckling mode $\hat{\phi}_{1}$ of the unperforated plate, determined by a linear FE-buckling analysis, is found by a multivariable regression on $S$ in the form of eight-order multivariate polynomials (see "Appendix B" and [18]). From this shape function, the distributions of $\Psi(x, y), \Sigma(x, y)=\phi_{, y}{ }^{2}-2 \phi_{, x} \phi_{, y}$, and $\hat{\gamma}(x, y)$ are deduced; see Figs. 14, 15, and 16. The buckled plate with two appropriately placed cutouts is shown in Fig. 17. Nearly $16 \%$ increase of the critical load multiplier and more than $5 \%$ mass saving could be achieved by these two holes. However, placing the cutouts as shown by the white dashed circles in Fig. 16, leads to a reduction of the critical load multiplier by $29.2 \%$ in comparison with the plate without cutouts.

\section{Some further notes}

Although in the demonstrative examples described above only circular cutouts are considered, the shape of the cutouts can be quite arbitrary. For instance, introducing in the plate used in Sect. 4.1 square cutouts instead of circular ones of similar size, leads to quite the same effect regarding the bifurcation load; see Fig. 18. 


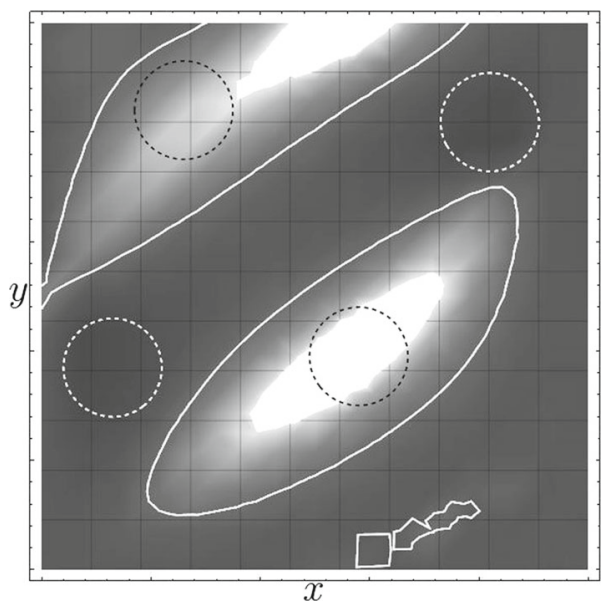

Fig. 16 Square plate, general loading case; $\hat{\gamma}(x, y)$

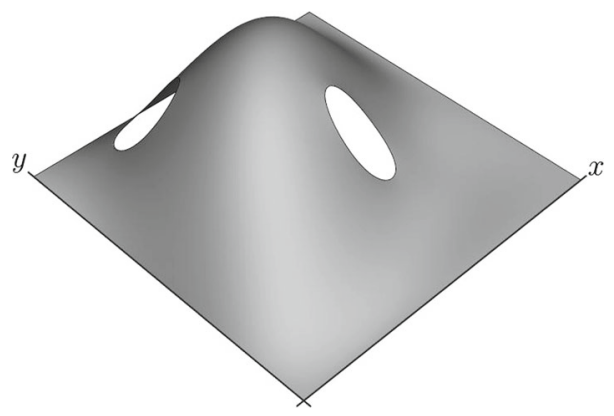

Fig. 17 Square plate with two adequate holes, general loading case: $\varphi=1.157$

Maybe one asks the question if cutouts, which are beneficial with respect to increased stability strength, might bring disadvantageous effects on the post-buckling behavior or on the influence of imperfections. In order to allay such fears, nonlinear FE analysis for studying the post-buckling and imperfection sensitivity behavior was performed for the above-mentioned plate with square cutouts, using as numerical example a plate with dimensions of $500 \times 500 \times 1$ length units. A very small transversal force in the plate's midpoint acted as load imperfection. Three intensities of this load imperfection are considered for both the unperforated and the perforated plate. The computed load-transversal displacement curves are presented in Fig. 18. There, $\tilde{\varphi}$ is the ratio between the actual shear load of the considered plate and the critical shear load of the perfect plate without cutouts, and $\tilde{w}_{0}$ is the transversal, i.e., out of plane displacement of the plate's midpoint divided by the plate's thickness.

These results reveal that the plate with cutouts shows, like those without cutouts, a stable post-buckling behavior. Hence, in a strictly spoken sense, also the plate with cutouts is, like the unperforated plate, not at all imperfections sensitive, meaning that imperfections turn the instability problem to a pure stress problem. Neither bifurcation nor snap-through buckling appears as it is typical for structures which in the perfect configuration lose stability of the trivial equilibrium state by bifurcation with stable post-buckling behavior along the bifurcated nontrivial path.

It is important to bear in mind that the focus of this paper lies on the buckling behavior. Nevertheless, it should be mentioned that by the introduction of cutouts not only the plate's stability behavior is changed but also its membrane stiffness is reduced. Furthermore, stress concentrations appear. Thus, cutouts, which influence the buckling behavior in a beneficial way, may be disadvantageous with respect to other kinds of failure, which are not considered in this paper. 


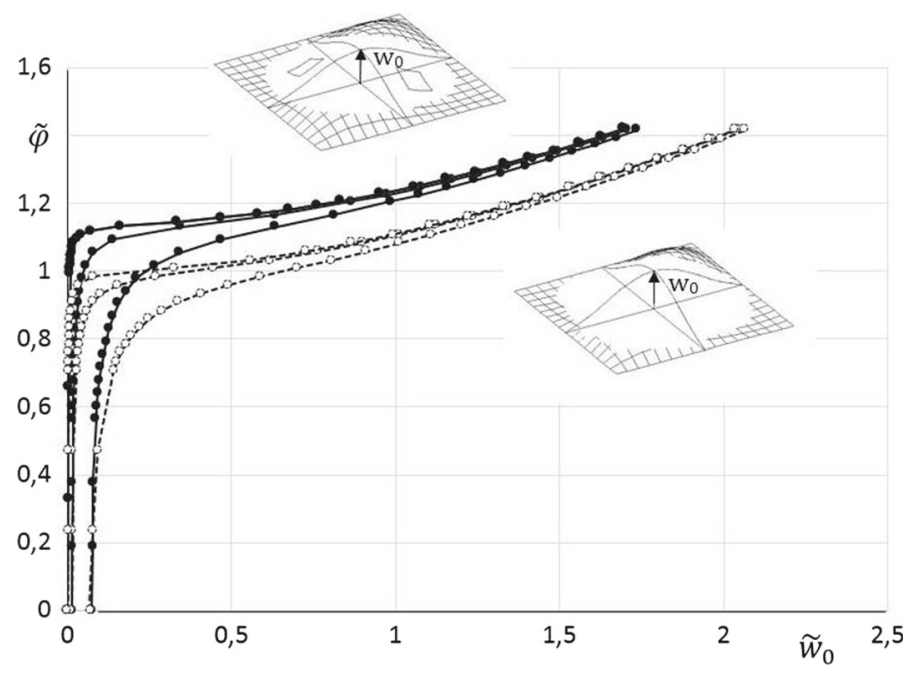

Fig. 18 Load displacement curves for the shear loaded square plate with different intensities of load imperfection; full lines: plate with cutouts; dotted lines: plate without cutouts

A further word of caution regards alternating loading: For instance, for shear loaded plates the beneficial effect of the appropriately positioned cutouts for a given direction of the shear loads will not only be lost when the load direction is reversed, but the cutouts will even substantially reduce the load-carrying capacity in terms of buckling when compared with that one of the unperforated plate. For instance, for the configuration with the two square cutouts, the critical load multiplier after load reversal is only $65 \%$ of that one before load reversal and is even $26 \%$ smaller than that of the unperforated plate! Such effects must be taken into account, when load reversal of the considered structure is a possible operating condition.

\section{Conclusions}

Criteria for possibilities of increasing the buckling resistance of thin plates, together with a reduction of structural mass by introduction of cutouts, are derived, and methodologies for achieving this advantageous goal in lightweight design are presented. Analytical as well as semi-analytical approaches are discussed and applied in demonstrative examples. It is shown that the advantageous character of the stable post-buckling behavior of plates does not diminish when cutouts are introduced, provided that these cutouts are not too large. Hence, imperfection sensitivity is not a question, too.

Some ideas for using the presented methodologies in optimization of plates for increased buckling resistance are given, which will be elaborated in future work. Moreover, the presented considerations provide hints for determining appropriate positions of openings, which, for some reasons, are required in flat structures in aerospace or naval architecture, when buckling resistance is a decisive design criterion.

\section{Acknowledgements Open access funding provided by TU Wien (TUW).}

Open Access This article is distributed under the terms of the Creative Commons Attribution 4.0 International License (http:// creativecommons.org/licenses/by/4.0/), which permits unrestricted use, distribution, and reproduction in any medium, provided you give appropriate credit to the original author(s) and the source, provide a link to the Creative Commons license, and indicate if changes were made.

\section{Appendix A}

In Remark 1 of the main body of this paper, it is mentioned that in the denominator of the Rayleigh quotient also for the perforated plate $n_{i j}(x, y)$, which due to the cutout(s) is inhomogeneously distributed over $S^{*}$, can be replaced by the uniformly distributed edge loadings $N_{i j}$. In this "Appendix A", this statement is justified by the following derivations. 
Provided that no self-equilibrating stresses (e.g., residual stresses, thermal stresses, etc.) exist, based on the derivation in [13], the Rayleigh quotient for estimating buckling load multipliers can be expressed by

$$
\lambda=\frac{\delta U}{\delta \bar{W}},
$$

with $\delta U$ being the strain energy due to a nontrivial out-of-plane displacement $\delta w(x, y)=\delta A \phi(x, y)$ with $\delta A \neq 0$. Note that $\delta U$ is not explicitly dependent on the external load! By $\delta \bar{W}$ the work of the external nominal loading in conjunction with the in-plane displacements of the loaded edges, caused by $\delta w$, is denoted.

Let us as an approximation, in analogy to the assumption of inextensibility of the beam axis when derivating buckling formulas for slender beams, assume that $\delta w(x, y)$ appears with an inextensible mid-surface of the plate. This model assumption implies that

1. the strain energy $\delta U$ caused by $\delta w$ consists of bending energy only;

2 . the change in the strains in the mid-surface, $\delta \varepsilon_{i j}^{0}$, caused by $\delta w$, is zero everywhere.

Implication (1.) leads to

$$
\begin{aligned}
\delta U & =\frac{1}{2} K \int_{S^{*}}\left[\Delta \delta w \Delta \delta w-2(1-v)\left(\delta w_{, x x} \delta w_{, y y}-\delta w_{, x y} \delta w_{, x y}\right] \mathrm{d} S\right. \\
& =\frac{(\delta A)^{2}}{2} K \int_{S^{*}}\left[\Delta \phi^{*} \Delta \phi^{*}-2(1-v)\left(\phi_{, x x}^{*} \phi_{, y y}^{*}-\phi_{, x y}^{*} \phi_{, x y}^{*}\right] \mathrm{d} S,\right.
\end{aligned}
$$

and implication (2.) stipulates

$$
\delta \varepsilon_{i j}^{0}=\frac{1}{2}\left(\delta u_{i, j}+\delta u_{j, i}\right)+\frac{1}{2} \delta w_{, i} \delta w_{, j}=0 .
$$

Consider a rectangular plate with edge lengths $a$ in $x$ direction and $b$ in $y$ direction, respectively. The origin of the $x, y$ coordinate system is in the left lower edge of the plate. Without restriction of generality, assume that only one hole is cut into the plate. Along the interval $\left[x_{I}, x_{I I}\right]$, corresponding to the projection of the hole to the $x$-axis, the boundary of the hole cuts an interval $\left[y_{1}(x), y_{2}(x)\right]$ out from the interval $[0, \mathrm{~b}]$. The same way, along the interval $\left[y_{I}, y_{I I}\right]$, corresponding to the projection of the hole to the $y$-axis, the boundary of the hole cuts an interval $\left[x_{1}(y), x_{2}(y)\right]$ out from the interval $[0, \mathrm{a}]$. Let us define [hole $\left.y\right](x)=\left[y_{1}(x), y_{2}(x)\right]$ and $\left[\operatorname{hole}_{x}\right](y)=\left[x_{1}(y), x_{2}(y)\right]$. Furthermore, let us define $\left[\right.$ hole $\left._{y}\right](x)=0$ outside the interval $\left[x_{I}, x_{I I}\right]$, and $\left[\right.$ hole $\left._{x}\right](y)=0$ outside the interval $\left[y_{I}, y_{I I}\right]$.

The in-plane edge displacement $\delta u(y)$ in $x$ direction at a position $y$ along the edge $x=a$, relative to the corresponding point $y$ along the edge $x=0$ is found by integrating $\delta u_{, x}(y)$ along the line $y=$ const with the exception of $\left[\operatorname{hole}_{x}\right](y)$.

Since, according to Eq. (21), $\delta \varepsilon_{x x}^{0}=\delta u_{x, x}+\frac{1}{2} \delta w_{, x} \delta w_{, x}=0$, one obtains $\delta u_{, x}(y)=\delta u_{x, x}(y)=$ $-\frac{1}{2} \delta w_{, i}(x, y) \delta w_{, j}(x, y)$. Using now $\delta w=\delta A \phi^{*}$, the relative displacement in $x$ direction at the position $y$ along the edge $x=a$ is determined by

$$
\delta u(y)=-\frac{(\delta A)^{2}}{2}\left[\int_{0}^{a} \phi_{, x}^{*}(x, y) \phi_{, x}^{*}(x, y) \mathrm{d} x-\int_{\left[\operatorname{hole}_{x}\right](y)} \phi_{, x}^{*}(x, y) \phi_{, x}^{*}(x, y) \mathrm{d} x\right] .
$$

Analogously, the relative displacement in $y$ direction along the edge $y=b$, i.e., $\delta v(x)$ is determined by

$$
\delta v(x)=-\frac{(\delta A)^{2}}{2}\left[\int_{0}^{b} \phi_{, y}^{*} \phi_{, y}^{*} \mathrm{~d} y-\int_{\left[\operatorname{hole}_{y}\right](x)} \phi_{, y}^{*} \phi_{, y}^{*} \mathrm{~d} y\right] .
$$

Eventually, the relative edge displacements due to shearing $\delta s_{y}(y)$ along the edge $x=a$ in the direction $y$, as well as $\delta s_{x}(x)$ along the edge $y=b$ in the direction $x$ are determined by

$$
\begin{aligned}
& \delta s_{y}(y)=-\frac{(\delta A)^{2}}{2}\left[\int_{0}^{a} \phi_{, x}^{*} \phi_{, y}^{*} \mathrm{~d} x-\int_{\left[\mathrm{hole}_{x}\right](y)} \phi_{, x}^{*} \phi_{, y}^{*} \mathrm{~d} x\right] \\
& \delta s_{x}(x)=-\frac{(\delta A)^{2}}{2}\left[\int_{0}^{b} \phi_{, y}^{*} \phi_{, x}^{*} \mathrm{~d} y-\int_{\left[\mathrm{hole}_{y}\right](x)} \phi_{, y}^{*} \phi_{, x}^{*} \mathrm{~d} y\right] .
\end{aligned}
$$


Assuming that only uniformly distributed in-plane edge loads are acting and taking the sign convention for these loads, as used in Eq. (1), into account, the work $\delta \bar{W}$ of the external loading, performed because of the appearance of $\delta w$, can now be expressed by

$$
\delta \bar{W}=\frac{(\delta A)^{2}}{2}\left[\int_{0}^{b} \int_{0}^{a} N_{i j} \phi_{, i}^{*} \phi_{, j}^{*} \mathrm{~d} x \mathrm{~d} y-\int_{\left[\operatorname{hole}_{y}\right](x)} \int_{\left[\operatorname{hole}_{x}\right](y)} N_{i j} \phi_{, i}^{*} \phi_{, j}^{*} \mathrm{~d} x \mathrm{~d} y\right] .
$$

Equation (22) can be reformulated as

$$
\delta \bar{W}=\frac{(\delta A)^{2}}{2}\left[\int_{S} N_{i j} \phi_{, i}^{*} \phi_{, j}^{*} \mathrm{~d} S-\int_{S^{* *}} N_{i j} \phi_{, i}^{*} \phi_{, j}^{*} \mathrm{~d} S\right]=\int_{S^{*}} N_{i j} \phi_{, i}^{*} \phi_{, j}^{*} \mathrm{~d} S .
$$

Here, it is assumed that all edges are movable in the $x-y$ plane and immovable in $z$ direction. Equations (20) and (23) put into Eq. (19) give

$$
\lambda^{*}=\frac{K \int_{S^{*}}\left[\Delta \phi^{*} \Delta \phi^{*}-2(1-v)\left(\phi_{, x x}^{*} \phi_{, y y}^{*}-\phi_{, x y}^{*} \phi_{, x y}^{*}\right] \mathrm{d} S\right.}{\int_{S^{*}} N_{i j} \phi_{, i}^{*} \phi_{, j}^{*} \mathrm{~d} S},
$$

as it is stated in Remark 1 of the main body of this paper.

Although the membrane force distribution $n_{i j}^{*}(x, y)$, which, in general, is inhomogeneously distributed over $S^{*}$, does not explicitly appear in Eq. (24), it enters the Rayleigh quotient implicitly via the buckling mode $\hat{\phi}^{*}$, approximated by $\phi^{*}$, which, for sufficiently large cutouts, is substantially different from $\hat{\phi}$. Needless to mention, that the integration domain is no longer $S$ but the mid-surface of the perforated plate, i.e., $S^{*}$.

\section{Appendix B}

In this "Appendix", for the sake of completeness, the function $\phi(x, y)$ approximating the buckling mode for the more general case presented in the Sect. 4.2 is provided. This function was determined for a square plate of edge length $a=500$ length units. Although the numbers given in the Tables below are only valid for the particular plate with this edge length, the criteria obtained with this function (see Fig. 16) hold for any square plate with equivalent edge load combinations and boundary conditions.

The multivariable polynomial approximating $\phi(x, y)$ for this case was determined by a multivariable regression on $S$ for the mode shape resulting from the eigenvector obtained from a linear FE-buckling analysis. The coefficients $C_{i}$ of the multivariable polynomial were obtained via least squares.

Multivariate polynomials seem to be a suitable type of functions for the implementation of the algorithm presented in Sect. 4. However, other types of functions, such as, e.g., Fourier-type functions may be used, too, for approximating $\phi(x, y)$. In the case under consideration, the approximate function $\phi(x, y)$ reads:

$$
\phi(x, y)=\sum_{i=1}^{45} C_{i} \cdot x^{I_{i, 1}} y^{I_{i, 2}}
$$

with the coefficients $C_{i}, I_{i, 1}$, and $I_{i, 2}$ given by Tables 1 and 2 . 
Table 1 Coefficients $C_{i}$ in Eq. (25)

\begin{tabular}{llll}
\hline Row number $i$ & & & \\
\hline 1 & $2.10784463536925 \times 10^{-21}$ & 24 & $-2.91540409432208 \times 10^{-9}$ \\
2 & $1.96235522653274 \times 10^{-21}$ & 25 & $8.91965180068485 \times 10^{-12}$ \\
3 & $-5.56356810835957 \times 10^{-18}$ & 26 & $-2.21074058759685 \times 10^{-14}$ \\
4 & $5.71635059484697 \times 10^{-15}$ & 27 & $2.19252755040569 \times 10^{-17}$ \\
5 & $-8.40754948977977 \times 10^{-18}$ & 28 & $9.59495404359974 \times 10^{-21}$ \\
6 & $1.33045163866146 \times 10^{-20}$ & 29 & $-2-2373168587741 \times 10^{-4}$ \\
7 & $-2.91945112221888 \times 10^{-12}$ & 30 & $-2.93661914218581 \times 10^{-6}$ \\
8 & $1.05748868993601 \times 10^{-14}$ & 31 & $1.81921257085803 \times 10^{-7}$ \\
9 & $7.17942880451076 \times 10^{-18}$ & 32 & $-1.39669116705207 \times 10^{-9}$ \\
10 & $-3.02497332814139 \times 10^{-20}$ & 33 & $2.62992736726934 \times 10^{-12}$ \\
11 & $8.18679356920937 \times 10^{-10}$ & 34 & $2.98318139815222 \times 10^{-15}$ \\
12 & $-3.46637445081927 \times 10^{-12}$ & 35 & $-9.57034680572213 \times 10^{-18}$ \\
13 & $-1.48260059451299 \times 10^{-14}$ & 36 & $2.44885312321049 \times 10^{-21}$ \\
14 & $4.08748706459427 \times 10^{-17}$ & $-4.26853962686435 \times 10^{-4}$ \\
15 & $1.43590858256316 \times 10^{-20}$ & 38 & $1.1907903675576 \times 10^{-4}$ \\
16 & $-1.25732981854897 \times 10^{-7}$ & 39 & $-3.06408062368617 \times 10^{-7}$ \\
17 & $-7.9154673404196 \times 10^{-10}$ & 40 & $-3.29973950193566 \times 10^{-8}$ \\
18 & $1.25051615041631 \times 10^{-11}$ & 41 & $3.42654271158376 \times 10^{-10}$ \\
19 & $-2.40956713096887 \times 10^{-14}$ & 42 & $-1.34692530265456 \times 10^{-12}$ \\
20 & $2.15447684286409 \times 10^{-17}$ & 43 & $2.54932176725693 \times 10^{-15}$ \\
21 & $-4.19239852671553 \times 10^{-20}$ & 44 & $-2.48447863741502 \times 10^{-18}$ \\
22 & $9.34888721155927 \times 10^{-6}$ & 45 & $1.1405637150238 \times 10^{-21}$ \\
23 & $4.11893057551076 \times 10^{-7}$ & & \\
\hline & & &
\end{tabular}

Table $2 x$ and $y$ exponents $I_{i, 1}$ and $I_{i, 2}$, respectively, in Eq. (25)

\begin{tabular}{|c|c|c|c|c|c|}
\hline Row number $i$ & Column 1 & Column 2 & & Column 1 & Column 2 \\
\hline 1 & 1 & 7 & 24 & 3 & 2 \\
\hline 2 & 0 & 8 & 25 & 3 & 2 \\
\hline 3 & 0 & 7 & 26 & 4 & 2 \\
\hline 4 & 0 & 6 & 27 & 5 & 2 \\
\hline 5 & 1 & 6 & 28 & 6 & 2 \\
\hline 6 & 2 & 6 & 29 & 0 & 1 \\
\hline 7 & 0 & 5 & 30 & 1 & 1 \\
\hline 8 & 1 & 5 & 31 & 2 & 1 \\
\hline 9 & 3 & 5 & 32 & 3 & 1 \\
\hline 10 & 0 & 4 & 33 & 4 & 1 \\
\hline 11 & 1 & 4 & 34 & 5 & 1 \\
\hline 12 & 2 & 4 & 35 & 6 & 1 \\
\hline 13 & 3 & 4 & 36 & 7 & 1 \\
\hline 14 & 4 & 4 & 37 & 0 & 0 \\
\hline 15 & 0 & 3 & 38 & 1 & 0 \\
\hline 16 & 1 & 3 & 39 & 2 & 0 \\
\hline 17 & 2 & 3 & 40 & 3 & 0 \\
\hline 18 & 3 & 3 & 41 & 4 & 0 \\
\hline 19 & 4 & 3 & 42 & 5 & 0 \\
\hline 20 & 5 & 3 & 43 & 6 & 0 \\
\hline 21 & 0 & 2 & 44 & 7 & 0 \\
\hline 22 & 1 & 2 & 45 & 8 & 0 \\
\hline 23 & 2 & 2 & & & \\
\hline
\end{tabular}




\section{References}

1. Megson, T.H.G.: Aircraft Structures, 3rd edn, pp. 406-425. Arnold, London (1999)

2. Wiedemann, J.: Leichtbau, pp. 78-79. Springer, Berlin (2007)

3. Brown, C.J., Yettram, A.L., Burnett, M.: Stability of plates with rectangular holes. J. Struct. Eng. 113, 1111-1116 (1987)

4. Onkar, A.K., Upadhyay, C.S., Yadav, D.: Stochastic finite element buckling analysis of laminated plates with circular cutout under uniaxial compression. J. Appl. Mech. 74, 798-809 (2006)

5. El-Sawy, K.M., Martini, M.I.: Elastic stability of bi-axially loaded rectangular plates with a single circular hole. Thin-Walled Struct. 45, 122-133 (2007)

6. Komur, M.A., Sonme, M.: Elastic buckling of rectangular plates under linearly varying in-plane normal load with a circular cutout. Mech. Res. Commun. 35, 361-371 (2008)

7. Maiorana, E., Pellegrino, C., Modena, C.: Elastic stability of plates with circular and rectangular holes subjected to axial compression and bending moment. Thin-Walled Struct. 47, 241-255 (2009)

8. Pham, C.H.: Shear buckling of plates and thin-walled channel sections with holes. J. Constr. Steel Res. 128, 800-811 (2017)

9. Chow, F.-Y., Narayanan, R.: Buckling of plates containing openings. In: Proceedings of the 7tn International Special Conference on Cold-Formed Steel Structures, Missouri University of Science and Technology (1984)

10. Rammerstorfer, F.G.: Buckling of elastic structures under tensile loads. Acta Mech. 229, 881-900 (2018)

11. Bitsche, R.D., Rammerstorfer, F.G.: Stress singularities at interfaces in structures: design charts. In: Yang Y.B., Leu L.J., Chen C.S., Su P.C. (eds.) Proceedings of the 11th East Asia-Pacific Conference on Structural Engineering and Construction. National Taiwan University, Paper-Nr. B08-01 (2008)

12. Ziegler, F.: Technische Mechanik der festen und flüssigen Körper, vol. 378. Springer, Wien (1985)

13. Bažant, Z.P., Cedolin, L.: Stability of Structures: Elastic, Inelastic, Fracture and Damage Theories, p. 323. World Scientific Publishing Co. Pte. Ltd., Singapore (2010)

14. Mang, H., Hofstetter, G.: Festigkeitslehre, p. 406. Springer, Wien (2000)

15. Leipholz, H.: Stability of elastic systems, p. 126. Sijthoff and Noordhoff, Alphen aan den Rijn (1980)

16. Wierzbicki, T.: Plates and shells. MIT Lecture Notes 2.081J/16.230J. MIT Library, Cambridge (2006)

17. Rammerstorfer, F.G.: Increase of the first natural frequency and buckling load of plates by optimal fields of initial stresses. Acta Mech. 27, 217-238 (1977)

18. Gasca, M., Sauer, T.: Polynomial interpolation in several variables. Adv. Comput. Math. 12, 377 (2000)

Publisher's Note Springer Nature remains neutral with regard to jurisdictional claims in published maps and institutional affiliations. 